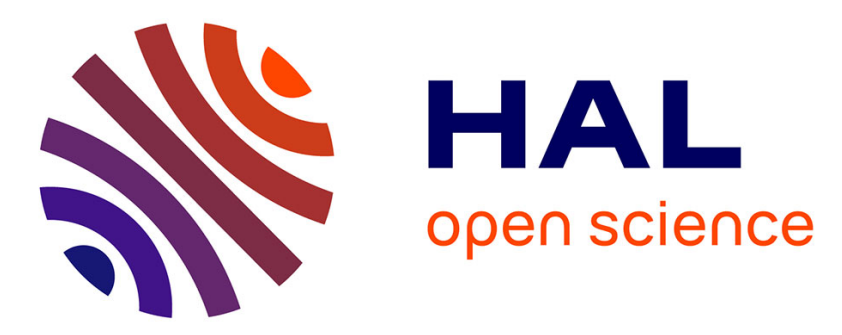

\title{
Viscoelastic impact between a cylindrical striker and a long cylindrical bar
}

\author{
M.-N. M.-N. Bussac, P. Collet, G. Gary, B. Lundberg, S. Mousavi
}

\section{To cite this version:}

M.-N. M.-N. Bussac, P. Collet, G. Gary, B. Lundberg, S. Mousavi. Viscoelastic impact between a cylindrical striker and a long cylindrical bar. International Journal of Impact Engineering, 2008, 35

(4), pp.226-239. 10.1016/j.ijimpeng.2007.02.003 . hal-00499098

\section{HAL Id: hal-00499098 \\ https://hal.science/hal-00499098}

Submitted on 9 Jul 2010

HAL is a multi-disciplinary open access archive for the deposit and dissemination of scientific research documents, whether they are published or not. The documents may come from teaching and research institutions in France or abroad, or from public or private research centers.
L'archive ouverte pluridisciplinaire $\mathbf{H A L}$, est destinée au dépôt et à la diffusion de documents scientifiques de niveau recherche, publiés ou non, émanant des établissements d'enseignement et de recherche français ou étrangers, des laboratoires publics ou privés. 


\section{Author's Accepted Manuscript}

Viscoelastic impact between a cylindrical striker and a long cylindrical bar

M.-N. Bussac, P. Collet, G. Gary, B. Lundberg, S. Mousavi

PII: S0734-743X(07)00016-4

DOI: doi:10.1016/j.ijimpeng.2007.02.003

Reference: IE 1462

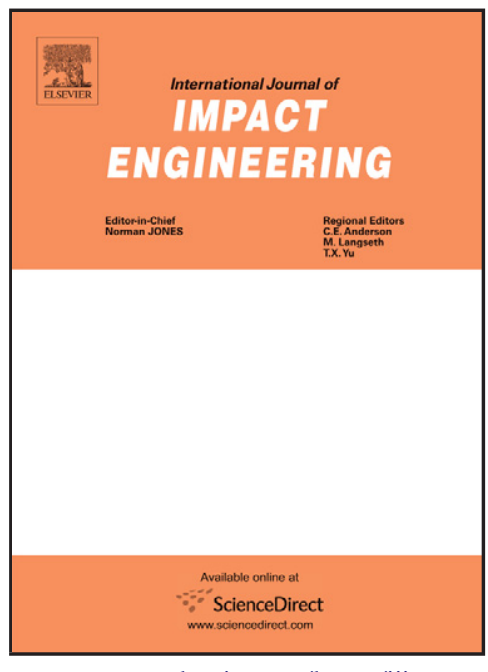

www.elsevier.com/locate/ijimpeng

To appear in: $\quad$ International Journal of Impact

Received date: 24 March 2006

Accepted date: $\quad 5$ February 2007

Cite this article as: M.-N. Bussac, P. Collet, G. Gary, B. Lundberg and S. Mousavi, Viscoelastic impact between a cylindrical striker and a long cylindrical bar, International Journal of Impact (2007), doi:10.1016/j.ijimpeng.2007.02.003

This is a PDF file of an unedited manuscript that has been accepted for publication. As a service to our customers we are providing this early version of the manuscript. The manuscript will undergo copyediting, typesetting, and review of the resulting galley proof before it is published in its final citable form. Please note that during the production process errors may be discovered which could affect the content, and all legal disclaimers that apply to the journal pertain. 


\title{
Viscoelastic impact between a cylindrical striker
}

\section{and a long cylindrical bar}

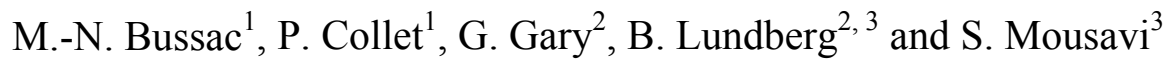 \\ ${ }^{1}$ Centre de Physique Théorique, CNRS UMR 7644, Ecole Polytechnique, 91128 Palaiseau, France \\ ${ }^{2}$ Laboratoire de Mécanique des Solides, CNRS UMR 7649, Ecole Polytechnique, 91128 Palaiseau, France \\ ${ }^{3}$ The Ångström Laboratory, Uppsala University, Box 534, SE-751 21 Uppsala, Sweden
}




\begin{abstract}
Axial impact between a cylindrical striker of finite length and a long cylindrical bar, both of linearly viscoelastic materials, is considered. General results are derived for the impact force, the particle velocity and the strain in the bar in terms of closed-contour integrals. Such results are derived also for the transfer of momentum and energy from the striker to the bar. Numerical results for a striker and a bar made of the same material but with different cross-sectional areas are compared. In viscoelastic impact, unlike elastic impact, the duration of contact may be finite and larger than two transit times for a wave front through the striker due to the formation of a tail of finite length after the main pulse. Furthermore, multiple contacts and separations of the striker and the bar may occur within a range of striker-to-bar characteristic impedance ratios. In the case of viscoelastic impact studied numerically, the duration of contact is at least as long and the momentum and energy transferred are at most as large as in elastic impact. Strains measured at three locations of a polymethyl methacrylate (PMMA) bar impacted by strikers of the same material as the bar agree well with the theoretical results.
\end{abstract}

\title{
Key words
}

Impact, viscoelastic, elastic, striker, bar, force, momentum, energy 


\section{Notation}

\section{Roman}

A

cross-sectional area

$c$

wave speed $\left[=(E / \rho)^{1 / 2}\right]$

d diameter

E

modulus

F

impact force

$f$

frequency

G impulse response

$H \quad$ Heaviside's unit step function

L length

$m \quad$ mass

$N \quad$ normal force

$p$

momentum

$R$

reflection coefficient $[=(1-r) /(1+r)]$

$r$

striker-to-bar characteristic impedance ratio $\left[=Z_{1} / Z_{2}\right]$

$t$

time

V

impact velocity

$v$

particle velocity

$W \quad$ energy

$x$

axial coordinate 
Greek

wave propagation coefficient $[=i \omega / c]$

$\delta$

delta function

$\varepsilon$

strain

$\eta \quad$ viscosity

$\lambda \quad$ wave length

$\rho \quad$ density

$\omega$

angular frequency $[=2 \pi f]$

Subscripts

$0,1,2 \quad$ striker/bar interface, striker, bar
A, B, C
strain gauges
g
gauge
n
direction of decreasing $x$
$\mathrm{p}$
direction of increasing $x$; provisional 


$\begin{array}{ll}\text { Superscripts } & \\ \mathrm{c} & \text { creep } \\ \mathrm{d}, \mathrm{e} & \text { dissipative, elastic } \\ \text { nd } & \text { non-dimensional } \\ \mathrm{r} & \text { relaxation } \\ \text { ref } & \text { reference }\end{array}$




\section{Introduction}

Axial impact between flat-ended cylindrical elastic bars was considered in detail by Saint-Venant $[1,2]$ in the 1860 s. Some of his classical results can be found, e.g., in the text book on elasticity by Timoshenko [3] and in those on impact by Goldsmith [4] and Johnson [5]. The results are important for the understanding of the mechanics of impact and related phenomena, and they have been used extensively in applications such as split Hopkinson pressure bar (SHPB) testing of materials [6] and percussive drilling of rock $[7,8]$. In the former application, they are a guide for the choice of impact velocities, dimensions of strikers and bars, and positions of strain gauges, while in the latter they are a basis for the design of various parts of rock drilling systems.

The need of SHPB testing of polymers, biological tissues and other soft materials has led to the use of bars and strikers made of materials such as polymethyl methacrylate (PMMA) which have viscoelastic [9, 10] rather than elastic response. Early studies of impact involving bars of such materials were carried out by Lee and Kanter [11] and Morrison [12] in the 1950s. If in SHPB testing the striker and the bars are made of the same viscoelastic material and have the same cross-sectional area, the impact force generally consists of a main pulse with duration two transit times for the viscoelastic wave front through the striker followed by a tail $[13,14]$ which has no elastic counterpart. This tail may result in undesirable overlap of waves at the strain gauge positions. In order to choose a ratio of the striker-to-bar cross-sectional area that avoids tail formation and other problems, such as multiple contacts and separations between the striker and the input bar, and to make similar assessments in other applications, one needs 
basic results for viscoelastic impact corresponding to those which exist since long time for elastic impact. The aim of this study was to provide such results.

In Section 2, the governing one-dimensional (1D) equations for axial impact between a cylindrical striker of finite length and a semi-infinite bar will be formulated for arbitrary linearly viscoelastic materials of the striker and the bar. These equations will be solved in the frequency domain for the impact force, the normal force, the particle velocity and the strain in the bar. Also, the momentum and energy transferred from the striker to the bar will be determined. In Section 3, the results obtained will be specialized to elastic impact and viscoelastic impact with the three-parameter viscoelastic standard model for the material of the striker and the bar. Fourier transforms will be inverted, and the results for the impact force and related functions of time will be expressed in terms of closed-contour integrals in the complex plane which are suitable for numerical evaluation. Experimental impact tests with strikers and bars made of PMMA will be presented in Section 4. Numerical results for viscoelastic impact will be interpreted and compared with corresponding results for elastic impact and with experimental results in Section 5 .

\section{Theory}

\subsection{Model, governing relations and general solution}

Consider axial viscoelastic impact between a cylindrical striker of finite length and a semi-infinite cylindrical bar, both with flat ends, as shown in Fig. 1. The striker (subscript 
1) has length $L_{1}$ and cross-sectional area $A_{1}$, and its material is characterized by the complex modulus $E_{1}(\omega)$, where $\omega$ is the angular frequency, and the density $\rho_{1}$. The impact velocity is $V_{1}$. The bar (subscript 2) has cross-sectional area $A_{2}$, and its material is characterized by the complex modulus $E_{2}(\omega)$ and the density $\rho_{2}$. Before impact, the bar is at rest.

It is assumed that initially plane cross-sections remain plane, radial inertia can be neglected, stress is uni-axial and wave propagation is 1D. Such conditions prevail if the wave lengths of the predominating waves generated in the striker and bar are much larger than the striker and bar diameters, respectively [15]. Under these conditions, the wave motion in the striker and the bar is governed by the two differential equations $\partial \hat{N} / \partial x=$ $A \rho i \omega \hat{v}$ and $\partial \hat{v} / \partial x=i \omega \hat{N} / A E$, where $\hat{N}(x, \omega)$ is the normal force, $\hat{v}(x, \omega)$ is the particle velocity and $x$ is an axial co-ordinate with origin at the striker/bar interface as shown in the figure. The first equation is that of motion, and the second originates from the compatibility condition $\partial \hat{v} / \partial x=i \omega \hat{\varepsilon}$, where $\hat{\varepsilon}=\hat{\sigma} / E$ is the normal strain and $\hat{\sigma}=\hat{N} / A$ the normal stress. The general solution of the two governing differential equations is $\hat{N}=\hat{N}_{\mathrm{p}} e^{-\gamma x}+\hat{N}_{\mathrm{n}} e^{\gamma x}$ and $\hat{v}=(1 / Z)\left(-\hat{N}_{\mathrm{p}} e^{-\gamma x}+\hat{N}_{\mathrm{n}} e^{\gamma x}\right)$, where $\hat{N}_{\mathrm{p}}(\omega)$ and $\hat{N}_{\mathrm{n}}(\omega)$ are amplitudes at $x=0$ associated with waves travelling in the directions of increasing and decreasing $x$, respectively, $Z=A(E \rho)^{1 / 2}$ is the characteristic impedance, $\gamma=i \omega / c$ is the wave propagation coefficient and $c=(E / \rho)^{1 / 2}$ is the wave speed. Here, the definition and notation $\hat{f}(\omega)=\int_{-\infty}^{\infty} f(t) e^{-i \omega t} \mathrm{~d} t$ has been used for the Fourier 
transform of the function $f(t)$, where $t$ is time, and the indices 1 and 2 for the striker and the bar, respectively, have been left out. 


\subsection{Impact force}

Let the velocity responses of the impact faces of the striker and the bar to impulsive forces $\delta(t)$ be $G_{1}(t)$ and $G_{2}(t)$, respectively, with velocities and forces directed into the impact faces defined as positive. Also, assume provisionally that the impact faces of the striker and the bar remain in contact after their initial contact at time $t=0$. As a result of impact, then, the velocities of the impact faces become $v_{1}(t)=V_{1}-G_{1}(t) * F_{\mathrm{p}}(t)$ and $v_{2}(t)=G_{2}(t) * F_{\mathrm{p}}(t)$, respectively, where $F_{\mathrm{p}}(t)$ is a provisional impact force. As the convolutions $G_{1}(t) * F_{\mathrm{p}}(t)$ and $G_{2}(t) * F_{\mathrm{p}}(t)$ are zero for $t<0$, and the impact faces have common velocity $v_{1}(t)=v_{2}(t)$ for $t>0$, the provisional impact force can be determined from the integral equation $[16,17]$

$$
\left[G_{1}(t)+G_{2}(t)\right] * F_{\mathrm{p}}(t)=V_{1} H(t)
$$

where $H(t)$ is Heaviside's unit step function.

Fourier transformation of Eq. (1) gives

$$
\hat{F}_{\mathrm{p}}=\frac{V_{1}}{\mathrm{i} \omega} \frac{1}{\hat{G}_{1}+\hat{G}_{2}} .
$$


By using the conditions $\hat{N}\left(-L_{1}, \omega\right)=0, \hat{N}(0, \omega)=-\hat{F}_{1}(\omega)$ and $\hat{v}(0, \omega)=-\hat{G}_{1}(\omega) \hat{F}_{1}(\omega)$ for the striker and $\hat{N}(0, \omega)=-\hat{F}_{2}(\omega), \hat{v}(0, \omega)=\hat{G}_{2}(\omega) \hat{F}_{2}(\omega)$ and $\hat{N}_{\mathrm{n}}(\omega)=0$ for the bar one obtains the impulse responses

$$
\hat{G}_{1}=\frac{1}{Z_{1}} \frac{1+e^{-2 \gamma_{1} L_{1}}}{1-e^{-2 \gamma_{1} L_{1}}}, \quad \hat{G}_{2}=\frac{1}{Z_{2}} .
$$

Substitution of Eqs. (3) into Eq. (2) gives

$$
\hat{F}_{\mathrm{p}}=\frac{Z_{1} V_{1}}{2} \frac{1+R}{i \omega} \frac{1-e^{-2 \gamma_{1} L_{1}}}{1+R e^{-2 \gamma_{1} L_{1}}}
$$

where

$$
R=\frac{Z_{2}-Z_{1}}{Z_{2}+Z_{1}}=\frac{1-r}{1+r}
$$

is the reflection coefficient, related to normal force, for waves in the striker at the striker/bar interface and

$$
r=\frac{Z_{1}}{Z_{2}}
$$

is the striker-to-bar characteristic impedance ratio. 
Clearly, the provisional impact force $F_{\mathrm{p}}(t)$ is positive initially. If it remains nonnegative for all time, the actual impact force becomes $F_{0}(t)=F_{\mathrm{p}}(t)$. If, instead, it changes sign from positive to negative at some finite time $t=t_{0}$, separation occurs at this time and the actual impact force becomes

$$
F_{0}(t)=\left[1-H\left(t-t_{0}\right)\right] F_{\mathrm{p}}(t)
$$

provided that the contact between the striker and the bar is not re-established. With $t_{0}=\infty$, this expression applies also to the case that $F(t)$ remains non-negative for all time.

2.3. Normal force, particle velocity and strain in the bar

The conditions $\hat{N}_{\mathrm{n}}(\omega)=0$ and $\hat{N}(0, \omega)=-\hat{F}_{0}(\omega)$ give the normal force

$$
\hat{N}=-\hat{F}_{0} e^{-\gamma_{2} x}
$$

and the particle velocity

$$
\hat{v}=\frac{\hat{F}_{0}}{Z_{2}} e^{-\gamma_{2} x} .
$$


in the bar. In particular, the particle velocity of the impact interface $\hat{v}_{0}(\omega)=\hat{v}(0, \omega)$ is given by

$$
\hat{v}_{0}=\frac{\hat{F}_{0}}{Z_{2}} .
$$

The strain in the bar is given by

$$
\hat{\varepsilon}=\frac{\hat{F}_{0}}{Z_{2} c_{2}} e^{-\gamma_{2} x} .
$$

\subsection{Transfer of momentum and energy to the bar}

The momentum transferred to the bar is equal to the impulse of the impact force, i.e.

$$
p_{2}=\int_{-\infty}^{\infty} F_{0} \mathrm{~d} t .
$$

By use of the definition $\hat{F}_{0}(\omega)=\int_{-\infty}^{\infty} F_{0}(t) e^{-i \omega t} \mathrm{~d} t$, this momentum can also be expressed

$$
p_{2}=\hat{F}_{0}(0)
$$

In the case of no separation, Eqs. (4), (7), (13) and the assumption $\gamma_{1}(\omega) \rightarrow 0$ as $\omega \rightarrow 0$, give 


$$
p_{2}=p_{1}, \quad t_{0}=\infty
$$

where $p_{1}=m_{1} V$ is the impact momentum and $m_{1}=A_{1} L_{1} \rho_{1}$ the mass of the striker. Thus, in the absence of separation there is a complete transfer of the impact momentum of the striker to the bar.

The energy transferred to the bar is equal to the work

$$
W_{2}=\int_{-\infty}^{\infty} F_{0} v_{0} \mathrm{~d} t
$$

carried out by the impact force. By use of Parseval's relation $\int_{-\infty}^{\infty} \bar{f} g \mathrm{~d} t=(1 / 2 \pi) \int_{-\infty}^{\infty} \overline{\hat{f}} \hat{g} \mathrm{~d} \omega$ (where the 'bar' denotes complex conjugate) and Eq. (10), this energy can also be expressed

$$
W_{2}=\frac{1}{2 \pi} \int_{-\infty}^{\infty} \frac{\left|\hat{F}_{0}\right|^{2}}{Z_{2}} \mathrm{~d} \omega
$$

\section{Applications}

\subsection{Elastic impact: striker and bar of the same material}

First, the results of Section 2 will be applied to the classical problem of elastic impact between a striker and a bar which consist of the same linearly elastic material with 
Young's modulus $E^{\mathrm{e}}$ and density $\rho$. This special case of viscoelastic impact, will be used as a reference for the more general case of viscoelastic impact to be considered in Section 3.2. Here, the quantities $E_{1}=E_{2}=E^{\mathrm{e}}, \rho_{1}=\rho_{2}=\rho, Z_{1}=Z_{1}^{\mathrm{e}}=A_{1}\left(E^{\mathrm{e}} \rho\right)^{1 / 2}$, $Z_{2}=Z_{2}^{\mathrm{e}}=A_{2}\left(E^{\mathrm{e}} \rho\right)^{1 / 2}, c_{1}=c_{2}=c^{\mathrm{e}}=\left(E^{\mathrm{e}} / \rho\right)^{1 / 2}, r=Z_{1} / Z_{2}=A_{1} / A_{2}$ and $R=$ $(1-r) /(1+r)$ are real and constant, while the quantities $\gamma_{1}=\gamma_{2}=\gamma^{\mathrm{e}}=i \omega / c^{\mathrm{e}}$ are imaginary and proportional to the angular frequency.

Equation (4) gives

$$
\frac{\hat{F}_{\mathrm{p}}}{F_{1}^{\mathrm{e}}}=\frac{1+R}{i \omega} \sum_{n=0}^{\infty}(-R)^{n}\left[e^{-i \omega n t_{1}^{\mathrm{e}}}-e^{-i \omega(n+1) t_{1}^{\mathrm{e}}}\right]
$$

and, after inversion,

$$
\frac{F_{\mathrm{p}}}{F_{1}^{\mathrm{e}}}=(1+R) \sum_{n=0}^{\infty}(-R)^{n}\left\{H\left(t-n t_{1}^{\mathrm{e}}\right)-H\left[t-(n+1) t_{1}^{\mathrm{e}}\right]\right\},
$$

where

$$
t_{1}^{\mathrm{e}}=2 \frac{L_{1}}{c^{\mathrm{e}}}, \quad F_{1}^{\mathrm{e}}=\frac{1}{2} Z_{1}^{\mathrm{e}} V_{1} .
$$


If the characteristic impedance of the striker is higher than or equal to that of the bar $(1 \leq r<\infty,-1<R \leq 0), F_{\mathrm{p}}(t)$ is nonnegative for $t \geq 0$. Therefore, the striker and the bar do not separate $\left(t_{0}=\infty\right)$, and by Eqs. (7) and (18) the impact force becomes

$$
\frac{F_{0}}{F_{1}^{\mathrm{e}}}=(1+R) \sum_{n=0}^{\infty}(-R)^{n}\left\{H\left(t-n t_{1}^{\mathrm{e}}\right)-H\left[t-(n+1) t_{1}^{\mathrm{e}}\right]\right\}, \quad 1 \leq r<\infty .
$$

If, in particular, the striker and the bar have equal characteristic impedances $(r=1$, $R=0$ ), all terms except the first vanish, and the impact force becomes a rectangular pulse with amplitude $F_{1}^{\mathrm{e}}$ and duration $t_{1}^{\mathrm{e}}$.

If the characteristic impedance of the striker is lower than that of the bar $(0<r<1,0<R<1), F(t)$ given by Eq. (18) changes sign from positive to negative at $t_{0}=t_{1}^{\mathrm{e}}$. Therefore, separation occurs at this time, and by Eqs. (7) and (18) the impact force becomes

$$
\frac{F_{0}}{F_{1}^{\mathrm{e}}}=(1+R)\left[H(t)-H\left(t-t_{1}^{\mathrm{e}}\right)\right], \quad 0<r<1 .
$$

For $1 \leq r<\infty$, the momentum $p_{2}$ transferred from the striker to the bar is given by Eq. (14). For $0<r<1, p_{2}$ can be obtained by substituting Eq. (21) into Eq. (12). As a result, the momentum transferred from the striker to the bar is given by 


$$
\frac{p_{2}}{p_{1}}=1, \quad 1 \leq r<\infty, \quad \frac{p_{2}}{p_{1}}=\frac{2}{1+r}, \quad 0<r<1 .
$$

Thus, when the characteristic impedance of the striker is equal to that of the bar or higher $(1 \leq r<\infty)$, there is no rebound, and the impact momentum of the striker is transferred to the bar without loss or gain $\left(p_{2}=p_{1}\right)$. Else $(0<r<1)$, rebound occurs and the momentum received by the bar is larger than the impact momentum of the striker $\left(p_{1}<p_{2}<2 p_{1}\right)$.

Equations (10) and (15) give the energy transferred to the bar $W_{2}=$ $\left(1 / Z_{2}\right) \int_{-\infty}^{\infty} F_{0}^{2} d t$. Substitution of Eqs. (20) and (21) into this expression gives

$$
1 \frac{W_{2}}{W_{1}}=1, \quad 1 \leq r<\infty, \quad 1 \frac{W_{2}}{W_{1}}=\frac{4 r}{(1+r)^{2}}, \quad 0<r<1,
$$

where $W_{1}=(1 / 2) m_{1} V_{1}^{2}$ is the kinetic impact energy of the striker. Thus, when the characteristic impedance of the striker is equal to that of the bar or higher $(1 \leq r<\infty)$, there is no rebound and the impact energy of the striker is completely transferred to the bar $\left(W_{2}=W_{1}\right)$. Else $(0<r<1)$, rebound occurs, and the transfer of energy is only partial $\left(0<W_{2}<W_{1}\right)$ 


\subsection{Viscoelastic impact: striker and bar of the same material}

Next, the results of Section 2 will be applied to the problem of viscoelastic impact between a striker and a bar which consist of the same linearly viscoelastic material represented by the three-parameter viscoelastic standard model and with density $\rho$. For this material, the complex modulus is [18]

$$
E=E^{\mathrm{e}} \frac{E^{\mathrm{d}}+i \omega \eta^{\mathrm{d}}}{E^{\mathrm{e}}+E^{\mathrm{d}}+i \omega \eta^{\mathrm{d}}},
$$

where the constitutive parameters $E^{\mathrm{e}}, E^{\mathrm{d}}$ and $\eta^{\mathrm{d}}$ (which can be interpreted in terms of a spring $E^{\mathrm{e}}$ in series with a parallel combination of a spring $E^{\mathrm{d}}$ and a viscous damper $\eta^{\mathrm{d}}$ ) are real and positive. The parameter $E^{\mathrm{e}}$, which is the high-frequency limit of the complex modulus, represents the initial purely elastic response of the material, while the parameters $E^{\mathrm{d}}$ and $\eta^{\mathrm{d}}$ are related to the viscous and dissipative response of the material. In the analysis that follows, the complex modulus will be expressed as

$$
E=E^{\mathrm{e}} \frac{t^{\mathrm{r}}}{t^{\mathrm{c}}} \frac{1+i \omega t^{\mathrm{c}}}{1+i \omega t^{\mathrm{r}}},
$$

where the constitutive parameters $E^{\mathrm{d}}$ and $\eta^{\mathrm{d}}$ have been replaced by the relaxation and creep time parameters 


$$
t^{\mathrm{r}}=\frac{\eta^{\mathrm{d}}}{E^{\mathrm{e}}+E^{\mathrm{d}}}, \quad t^{\mathrm{c}}=\frac{\eta^{\mathrm{d}}}{E^{\mathrm{d}}} .
$$

These relations show that the viscoelastic model is close to the elastic model $E=E^{\mathrm{e}}$ at high frequencies where $\omega t^{\mathrm{r}}>>1$.

The quantities $\rho_{1}=\rho_{2}=\rho, r=Z_{1} / Z_{2}=A_{1} / A_{2}$ and $R=(1-r) /(1+r)$ are real and constant, while $E_{1}=E_{2}=E, Z_{1}=A_{1}(E \rho)^{1 / 2}, Z_{2}=A_{2}(E \rho)^{1 / 2}, c_{1}=c_{2}=c=$ $(E / \rho)^{1 / 2}$ and $\gamma_{1}=\gamma_{2}=\gamma=i \omega / c$ are complex-valued functions of frequency.

\subsection{Normalization}

In the remainder of Section 3, and for the presentation of numerical results in Section 5, dimensionless quantities will be introduced, which are normalized to reference quantities related to the striker (subscript 1) in elastic (superscript e) impact. Thus, the reference quantities will be chosen as $t^{\mathrm{ref}}=t_{1}^{\mathrm{e}}=2 L_{1} / c^{\mathrm{e}}$ for time, $\omega^{\mathrm{ref}}=1 / t^{\mathrm{ref}}=c^{\mathrm{e}} / 2 L_{1}$ for angular frequency, $c^{\text {ref }}=c^{\mathrm{e}}=\left(E^{\mathrm{e}} / \rho\right)^{1 / 2}$ for wave speed, $\gamma^{\text {ref }}=\omega^{\text {ref }} / c^{\text {ref }}=1 / 2 L_{1}$ for wave propagation coefficient, $Z^{\text {ref }}=Z_{1}^{\mathrm{e}}=A_{1}\left(E^{\mathrm{e}} \rho\right)^{1 / 2}$ for characteristic impedance, $v^{\text {ref }}=$ $(1 / 2) V_{1}$ (correspondingly, $\hat{v}^{\text {ref }}=v^{\text {ref }} t^{\text {ref }}$ ) for particle velocity, $F^{\text {ref }}=Z^{\text {ref }} v^{\text {ref }}=F_{1}^{\mathrm{e}}=$ $(1 / 2) Z_{1}^{\mathrm{e}} V_{1}$ (correspondingly, $\hat{F}^{\mathrm{ref}}=F^{\text {ref }} t^{\text {ref }}$ ) for impact force, $p^{\text {ref }}=F^{\text {ref }} t^{\text {ref }}=p_{1}=m_{1} V_{1}$ for momentum, and $W^{\text {ref }}=F^{\text {ref }} v^{\text {ref }} t^{\text {ref }}=W_{1}=(1 / 2) m_{1} V_{1}^{2}$ for energy. 
Substitution of $t=t^{\text {nd }} t^{\text {ref }}, \omega=\omega^{\text {nd }} \omega^{\text {ref }}, c=c^{\text {nd }} c^{\text {ref }}$, etc., and using $Z_{2}^{\text {nd }}=1 / r$, gives dimensionless expressions for impact force, momentum and energy. In particular, there is the dimensionless expression

$$
\hat{F}_{\mathrm{p}}=\frac{1+R}{\gamma} \frac{1-e^{-\gamma}}{1+R e^{-\gamma}}
$$

for the provisional impact force, where

$$
\gamma=\frac{i \omega}{c}, \quad c=\left(\frac{t^{\mathrm{r}}}{t^{\mathrm{c}}}\right)^{1 / 2}\left(\frac{1+i \omega t^{\mathrm{c}}}{1+i \omega t^{\mathrm{r}}}\right)^{1 / 2}
$$

are the dimensionless wave propagation coefficient and wave speed, respectively, with the properties $\gamma \rightarrow i \omega$ and $c(\omega) \rightarrow 1$ as $\omega \rightarrow \infty$, and the superscripts 'nd' indicating nondimensionality have been left out. By use of the same convention, Eq. (7) for the impact force $F_{0}$, Eq. (10) for the particle velocity $v_{0}$, Eqs. (12) and (13) for the momentum $p_{2}$, and Eqs. (15) and (16) for the energy $W_{2}$ remain the same in dimensionless form, while Eq. (14) becomes $p_{2}=1, t_{0}=\infty$.

In elastic impact, considered in Section 3.1, the dimensionless impact force $F_{0}(t)$, the momentum $p_{2}$ and the energy $W_{2}$ depend only on the striker-to-bar characteristic impedance ratio $r$. In the case of viscoelastic impact considered in Section 3.2, these quantities depend also on the dimensionless characteristic time parameters $t^{\mathrm{r}}$ and $t^{\mathrm{c}}$. In both cases $r=A_{1} / A_{2}$ as the material is assumed to be the same in the striker and the bar. 


\subsection{Inversion of the Fourier transform of the provisional impact force}

Inversion of Eq. (27) gives

$$
F_{\mathrm{p}}(t)=\frac{1+R}{2 \pi} \int \frac{1-e^{-\gamma(\omega)}}{1+R e^{-\gamma(\omega)}} e^{i \omega t} \frac{\mathrm{d} \omega}{\gamma(\omega)},
$$

where, by Eqs. (28),

$$
\gamma(\omega)=i \omega\left(\frac{t^{\mathrm{c}}}{t^{\mathrm{r}}}\right)^{1 / 2}\left(\frac{1+i \omega t^{\mathrm{r}}}{1+i \omega t^{\mathrm{c}}}\right)^{1 / 2} .
$$

In the evaluation of this integral, care has to be taken to ensure causality. The integration path is taken as a line in the lower complex half plane $\operatorname{Im}(\omega)<0$ parallel to the real axis. Also, in Eq. (30) the standard definition is taken for the square root in the complex plane, viz., with a cut on the real negative axis. With this definition, the function $\gamma(\omega)$ is analytic in the complex plane except for a cut along the segment $I=i\left[1 / t^{\mathrm{c}}, 1 / t^{\mathrm{r}}\right]$ of the imaginary axis. Moreover, $\gamma(\omega)=i \omega+O(1)$ for $\omega$ of large modulus. This implies that for $t<0$ the path of integration can be changed to any line in the lower half plane parallel to the real axis, and it follows that for such $t$ there is the result $F(t)=0$.

For $t>0$ it is observed that if $\operatorname{Im}(\omega)<0$, then $\operatorname{Re}(\gamma(\omega))>0$. Therefore, for $\omega$ in the lower half plane, there is the expansion 


$$
\frac{1-e^{-\gamma(\omega)}}{1+R e^{-\gamma(\omega)}}=1-(1+R) \sum_{n=1}^{\infty}(-R)^{n-1} e^{-n \gamma(\omega)}
$$

It can be verified that for any $\omega$ in the lower half plane this series is absolutely convergent. Consider now separately each integral

$$
I_{n}(t)=\int e^{i \omega t} e^{-n \gamma(\omega)} \frac{\mathrm{d} \omega}{\gamma(\omega)}
$$

From the behaviour $\gamma(\omega)=i \omega+O(1)$ for $\omega$ of large modulus, it follows that for $n>t$ the integration contour can be deformed to any line in the lower complex half plane, and for such $t$ the integral $I_{n}(t)=0$. This implies

$$
F_{\mathrm{p}}(t)=\frac{1+R}{2 \pi} \int\left[1-(1+R) \sum_{1 \leq n \leq t}(-R)^{n-1} e^{-n \gamma(\omega)}\right] e^{i \omega t} \frac{\mathrm{d} \omega}{\gamma(\omega)},
$$

where, for simplicity, it will be assumed that $t \neq n$. In this expression, the integration contour can now be deformed provided that the singularities of the integrand are not crossed. These singularities are the point $\omega=0$, where $\gamma$ vanishes, and the cut along the segment $I=i\left[1 / t^{\mathrm{c}}, 1 / t^{\mathrm{r}}\right]$. In particular, one obtains

$$
F_{\mathrm{p}}(t)=\frac{1+R}{2 \pi} \int_{C \cup \operatorname{Im}(\omega)=s}\left[1-(1+R) \sum_{1 \leq n \leq t}(-R)^{n-1} e^{-n \gamma(\omega)}\right] e^{i \omega t} \frac{\mathrm{d} \omega}{\gamma(\omega)}
$$


where $s>1 / t^{\mathrm{r}}$ and $C$ is a curve below the line $\operatorname{Im}(\omega)=s$ enclosing the point $\omega=0$ and the cut $i\left[1 / t^{\mathrm{c}}, 1 / t^{\mathrm{r}}\right]$. With arguments as above, it can be shown that the part of the integral on $\operatorname{Im}(\omega)=s$ does not depend on $s$ and is equal to zero. Therefore, the final result for the provisional impact force becomes

$$
F_{\mathrm{p}}(t)=\frac{1+R}{2 \pi} \int_{C}\left[1-(1+R) \sum_{1 \leq n \leq t}(-R)^{n-1} e^{-n \gamma(\omega)}\right] e^{i \omega t} \frac{\mathrm{d} \omega}{\gamma(\omega)}
$$

In the numerical evaluation of this result, the contour $C$ was taken as a circle with centre at $i\left(1 / t^{\mathrm{c}}+1 / t^{\mathrm{r}}\right)$ and radius $2 / t^{\mathrm{r}}$. The inversion procedure used is not limited to viscoelastic materials with complex modulus given by Eq. (25); it can be applied also to more general viscoelastic materials which have complex modulus $E(\omega)$ with adequate properties of analyticity.

The provisional velocity $v_{\mathrm{p}}(t)$ of the impact face, corresponding to $F_{\mathrm{p}}(t)$, was obtained on the basis of Eq. (10) by inversion of $\hat{v}_{\mathrm{p}}=\left(1 / Z_{2}\right) \hat{F}_{\mathrm{p}}=(r / c) \hat{F}_{\mathrm{p}}$ with $r, c$ and $\hat{F}_{\mathrm{p}}$ given by Eqs. (6), (28 b) and (27), respectively. Then, the impact force and the velocity of the impact face were obtained in the interval $0 \leq t<t_{0}$ as $F_{0}(t)=F_{\mathrm{p}}(t)$ and $v_{0}(t)=v_{\mathrm{p}}(t)$, respectively. The strain in the bar $\varepsilon(x, t)$ was evaluated similarly on the basis of Eq. (11).

The momentum $p_{2}$ and energy $W_{2}$ transferred to the bar were obtained from Eqs. (12) and (15), respectively, through numerical integration. In cases with separation of the striker and the bar at a finite time $t_{0}$, the integrations were carried out from zero to this 
time. Thus, (i) if separation does not occur, or (ii) if separation occurs once without reestablishment of contact, then $p_{2}$ and $W_{2}$ represent the total momentum and energy transferred to the bar through the impact process. (iii) If separation occurs and contact is re-established at least once, however, then $p_{2}$ and $W_{2}$ do not represent the total momentum and energy finally transferred to the bar through multiple impacts.

\section{Experiments}

Impact tests were carried out with the experimental set up shown in Fig. 2. Cylindrical strikers and a cylindrical bar were fabricated from a single bar of PMMA with density $1183 \mathrm{~kg} / \mathrm{m}^{3}$. The original bar was machined to a diameter of $11.93 \mathrm{~mm}$, which made strikers and bar fit into the barrel of an air gun with a tolerance of $0.06 \mathrm{~mm}$. The ends of the strikers and the bar were machined to be flat and perpendicular to the centre lines. The lengths of the strikers were 120.0,240.0 and $360.0 \mathrm{~mm}$, and that of the bar was 2000 $\mathrm{mm}$. The bar was supported by eight Teflon bearings mounted on the same heavy aluminium beam as the air gun.

The impact end of the bar was located inside the barrel about $40 \mathrm{~mm}$ from the muzzle. Near the muzzle, axial slots in the barrel allowed the air to escape on both sides

of the striker. In this way no significant air cushion was formed in front of the striker, and the pressure acting on the rear end of the striker during impact was negligible. The slots also made it possible to estimate the impact velocity from the time of flight of the striker between two axial positions. The time of flight was measured by means of a chronograph for measurement of the velocities of rifle bullets. It had been modified for measurement 
of lower velocities through reduction of the distance between its two photo-sensors to $30.5 \mathrm{~mm}$. The second of these was positioned at a distance of $36 \mathrm{~mm}$ from the bar end. The inaccuracy of the impact velocity obtained in this way is estimated to be within \pm 5 $\%$.

The bar was instrumented with pairs of diametrically opposite and axially oriented resistive strain gauges (GFLA-6-350-70-1) A, B and C at distances $x_{\mathrm{A}}=120, x_{\mathrm{B}}=420$ and $x_{\mathrm{C}}=720 \mathrm{~mm}$, respectively, from its impacted end. The active length of the strain gauges was $6 \mathrm{~mm}$, and they were connected to Wheatstone bridges followed by amplifiers (Measurement Group 2210B) with bandwidth $100 \mathrm{kHz}$ so as to make the output signals proportional to the symmetric components of strain. These signals were recorded with sampling frequency $1 \mathrm{MHz}$ by a 16-bit data acquisition board. Shunt calibration was used for each channel. The inaccuracy of the measured strains $\varepsilon_{\mathrm{A}}, \varepsilon_{\mathrm{B}}$ and $\varepsilon_{\mathrm{C}}$ is estimated to be within $\pm 1 \%$.

A separate impact test was carried out in order to identify the complex modulus of the PMMA material of the bar and the strikers. In this test, a standard lead projectile was fired with an air rifle against the impact end of the bar. The front of the projectile was semi-spherical, and the rear had the shape of a truncated cone. The projectile had diameter $4.5 \mathrm{~mm}$, length $5 \mathrm{~mm}$ and mass $0.4 \mathrm{~g}$. This choice of projectile was made in order to obtain sufficient excitation at higher frequencies. The strains $\varepsilon_{\mathrm{A}}$ and $\varepsilon_{\mathrm{C}}$ associated with the wave generated were measured as described.

In a first step, the complex modulus of the bar was identified non-parametrically as $E=-\rho(\omega / \gamma)^{2}$ with $\gamma=\ln \left(\hat{\varepsilon}_{\mathrm{A}} / \hat{\varepsilon}_{\mathrm{C}}\right) /\left(x_{\mathrm{C}}-x_{\mathrm{A}}\right)$ from the changes in amplitude and phase 
of a strain pulse travelling from $x_{\mathrm{A}}$ to $x_{\mathrm{C}}[19,20]$. In a second step, the constitutive parameters $E^{\mathrm{e}}, E^{\mathrm{d}}$ and $\eta^{\mathrm{d}}$ were obtained by minimizing the difference between the nonparametric complex modulus and the complex modulus given by Eq. (24) for the threeparameter viscoelastic standard model. This was done in the frequency interval 0.5 to 32 $\mathrm{kHz}$ in the sense of least squares. The same number of discrete frequencies were considered in each subinterval $0.5-1,1-2,2-4, \ldots$, and $16-32 \mathrm{kHz}$. The relaxation and creep time parameters $t^{\mathrm{r}}$ and $t^{\mathrm{c}}$ were determined from Eqs. (26).

The time scale $t^{\prime}$ of the recorded strains and the time scale $t$ used in the theoretical analysis are translated relative to each other by an amount which is not known a priori. Thus, the origin $t=0$ is defined as the initial instant of contact between the striker and the bar, while $t^{\prime}=0$ is not well defined in relation to the impact event. In order to compare the theoretical and experimental results for strains in the bar at A, B and $\mathrm{C}, t^{\prime}$ was related to $t$ through $t^{\prime}-t=t_{\mathrm{A}}^{\prime}-t_{\mathrm{A}}$, where $t_{\mathrm{A}}^{\prime}$ and $t_{\mathrm{A}}$ represent the arrival at $\mathrm{A}$ of a strain wave generated through impact. In this way, the recorded strains could be presented as functions of time $t$. The arrival time $t_{\mathrm{A}}^{\prime}$ was taken as the time at which the recorded strain at A had reached half of its peak value, while $t_{\mathrm{A}}$ was estimated as $x_{\mathrm{A}} / c^{\mathrm{e}}$, where $x_{\mathrm{A}}$ is the distance from the impacted end to A and $c^{\mathrm{e}}=\left(E^{\mathrm{e}} / \rho\right)^{1 / 2}$ is the theoretical speed of the discontinuous wave front. 


\section{Results and discussion}

\subsection{Theoretical results}

Numerical results for elastic and viscoelastic impact of a striker and a bar made of the same material are shown in Figs. 3 to 6. For the case of viscoelastic impact, the dimensionless relaxation and creep time parameters were chosen as $t^{\mathrm{r}}=0.50$ and $t^{\mathrm{c}}=0.75$ (somewhat smaller and larger, respectively, than the corresponding parameters 0.56 and 0.60 for the $120 \mathrm{~mm}$ PMMA striker, Section 5.3). As the double transit time for a wave front through the striker is $t_{1}^{\mathrm{e}}=1$, the time parameters characterizing the relaxation, creep and wave phenomena in the striker are related as $t^{\mathrm{r}}=0.50<t^{\mathrm{c}}=0.75<t_{1}^{\mathrm{e}}=1$, and they are all of the order of unity. Therefore the viscoelastic impact process can be expected to be close to its elastic counterpart at times $t<<1$, in the early wave formation process, when there has been little time for relaxation and creep to occur. Viscous effects can be expected to be significant at times $t$ of the order of unity or larger. In particular they are significant at the time $t=1$ when a wave front has propagated from the impact face to the free end of the striker and back, and at later times $t>1$. The importance of the relative magnitudes of the time considered and the time parameters representing viscous effects and wave propagation was thoroughly discussed by Lee and Kanter [11].

The impact forces $F(t)$ generated through elastic impact and viscoelastic impact are compared in Fig. 3 for different bar-to-striker characteristic impedance ratios $r$. The force $(1 / 2) Z_{1}^{\mathrm{e}} V_{1}$, generated through elastic impact between a striker and a bar with the 
same characteristic impedance $Z_{1}^{\mathrm{e}}$, and the double transit time $2 L_{1} / c^{\mathrm{e}}$ for a wave through the elastic striker are used as units.

The elastic and viscoelastic impact forces consist of a main pulse that is either isolated or followed by a tail. The initial amplitude $F_{0}(0+)=2 /(1+r)$ and the unit width of the elastic and viscoelastic main pulses are the same. This is because the amplitude and the speed of the discontinuous viscoelastic wave front depend on the parameter $E^{\mathrm{e}}$, representing the initial elastic response of the viscoelastic material, but not on the relaxation and creep time parameters $t^{\mathrm{r}}$ and $t^{\mathrm{c}}$. Thus, the elastic and viscoelastic wave fronts have the same speed $c=1$. Equation (28 b) shows that for the viscoelastic waves this is the high-frequency limit of the phase velocity which is approached at frequencies $\omega>>1 / t^{\mathrm{r}}$. The decay in amplitude of the viscoelastic main pulse in the interval $0<t<1$ below the constant level of its elastic counterpart, is due to stress relaxation. In accordance with the discussion above, this decay is insignificant at times $t<<1$ but significant at time $t=1$.

In elastic impact, the formation of a tail for $t>1$, after the main pulse, is due to the multiple reflections of waves which take place between the ends of the striker when the characteristic impedance of the striker is larger than that of the bar so that $r>1$. This results in a tail which decays discontinuously through jumps at $t=1,2,3, \ldots(r=1.2$ and 2.0 in Fig. 3). In viscoelastic impact, the tail formation may be due to stress relaxation alone which produces a tail which decays continuously ( $r=0.8,0.9$ and 1.0 in Fig. 3). It may also be due to the combined effect of impedance mismatch and stress relaxation, which gives rise to a tail which decays in both ways ( $r=1.2$ and 2.0 in Fig. 3). 
The dependence on $r$ of the duration of contact $t_{0}$ (till the instant of first separation, if applicable) is compared for elastic impact and the case of viscoelastic impact in Fig. 4. In elastic impact, the duration of contact jumps from $t_{0}=1$ for $r<1$ to $t_{0}=\infty$ for $r \geq 1$. In the case of viscoelastic impact, $t_{0}$ increases from $t_{0}=1$ for $r<0.692$ to $t_{0}=2$ for $r=0.915$. Then, at this value of $r$, the duration of contact jumps from $t_{0}=2$ to $t_{0}=\infty$. Thus, the duration of contact in the case of viscoelastic impact is at least as long as that in elastic impact. "Jump", as a verb or a noun, is used here and below even though the existence of discontinuities has not been proved.

The dependence on $r$ of the momentum $p_{2}$ transferred from the striker to the bar (till the time of first separation, if applicable) is compared for elastic impact and the case of viscoelastic impact in Fig 5, where the impact momentum $m_{1} V_{1}$ of the striker is used as unit. The remaining momentum of the striker after this transfer is $1-p_{2}$.

In elastic impact, the momentum transferred decreases with increasing $r$ as $p_{2}=2 /(1+r)>1$ for $r<1$ and is constant, $p_{2}=1$, for $r \geq 1$. Thus, the remaining momentum of the elastic striker is (i) negative for $r<1 \quad(r=0.5,0.8$ and 0.9 in Fig. 3) and (ii) zero for $r \geq 1(r=1,1.2$ and 2 in Fig. 3). In the case of viscoelastic impact, the momentum transferred decreases from a value in the interval $1<p_{2}<2$ for $r=0$ to $p_{2}=1$ for $r=0.830$ and further to a minimum value $p_{2}=0.982$ for $r=0.915$. Then, at this value of $r$, the momentum transferred jumps to the level $p_{2}=1$ which remains constant for $r>0.915$. Thus, the remaining momentum of the viscoelastic striker is (i) negative for $r<0.830$, (ii) positive for $0.830<r<0.915$ and (iii) zero for $r>0.915$. In the first case ( $r=0.5$ and 0.8 in Fig. 3), the striker rebounds and most likely the contact 
with the bar is not re-established, while in the third case ( $r=1,1.2$ and 2 in Fig. 3) the striker, after full transfer of its momentum, stays in forceless contact with the bar. In the second case ( $r=0.9$ in Fig. 3 ), the striker has a positive momentum after its separation from the bar. This means that contact between the striker and the bar will be reestablished and in this way additional momentum will be transferred to the bar. Possibly, separation and re-establishment of contact may occur for a number of times until $p_{2} \geq 1$ so that, finally, the remaining momentum of the striker becomes zero or negative. It is seen that the momentum transferred from the striker to the bar through viscoelastic impact is at most as large as that transferred through elastic impact.

The dependence on $r$ of the energy $W_{2}$ transferred from the striker to the bar is compared for elastic impact and the case of viscoelastic impact in Fig 6, where the impact energy $(1 / 2) m_{1} V_{1}^{2}$ of the striker is used as unit. In elastic impact, the energy transferred consists of equal parts of elastic strain energy and kinetic energy which remain constant. It increases with increasing $r$ as $W_{2}=4 r /(1+r)^{2}$ for $r<1$ and is constant, $W_{2}=1$, for $r \geq 1$. In the case of viscoelastic impact, all energy transferred is finally dissipated as heat. This energy increases with increasing $r$ and approaches 1 for large values of $r$. At $r=0.915$ there is a jump in $W_{2}$ from 0.9065 to 0.9071 . It is seen that the energy transferred from the striker to the bar through viscoelastic impact is at most as large as that transferred through elastic impact.

For the case of viscoelastic impact, some significant values and intervals of $r$ can be summarised as follows: (i) For $r<0.692$, the impact force consists of a main pulse alone and $t_{0}=1$. The striker rebounds and most likely the contact with the bar is not reestablished. (ii) For $0.692<r<0.830$, the impact force has a tail of finite length and 
$1<t_{0}<1.462$. Again, the striker rebounds and most likely the contact with the bar is not re-established. (iii) For $0.830<r<0.915$, the impact force has a tail of finite length and $1.462<t_{0}<2$. In this case, contact is re-established at least once before final separation. (iv) For $r=0.915$, there is a jump in the duration of contact from $t_{0}=2$ to $\infty$, in the momentum transferred from $p_{2}=0.982$ to 1 , and in the energy transferred from $W_{2}=0.9065$ to 0.9071 . (v) For $r>0.915$, the impact force has a tail of infinite length and the duration of impact is infinite.

For $0.692<r \leq 1$, the amplitude of the tail decays continuously with increasing time. For $r>1$, there are also step-wise drops in amplitude at integral values of time due to the decrease in cross-sectional area from the striker to the bar and the multiple reflections of waves within the striker.

The general character of the results obtained is not limited to viscoelastic materials with complex modulus given by Eq. (25); similar results can be obtained for more general viscoelastic materials which have complex modulus $E(\omega)$ with the same properties of analyticity. It follows from the results that in SHPB testing, with striker and bars made of the same viscoelastic material, the tail of the impact force and the phenomenon of multiple impacts can be avoided by choosing $r$ sufficiently small. For the hypothetic material considered here, the critical value of $r$ in this respect is 0.692 which corresponds to a striker-to-bar diameter ratio of 0.832 .

\subsection{Identification of PMMA}


The non-parametric and parametric results for the complex modulus $E$ of the PMMA material of the bar and the strikers are shown versus frequency $f=2 \pi / \omega$ in Fig. 7, where also the discrete points used for parametric identification are indicated. The constitutive parameters of the three-parameter linearly viscoelastic solid model were estimated to be $E^{\mathrm{e}}=5.78 \mathrm{GPa}, E^{\mathrm{d}}=72.3 \mathrm{GPa}$ and $\eta^{\mathrm{d}}=4.75 \mathrm{MPas}$. From these parameters and Eqs. (26), the relaxation and creep time parameters were obtained as $t^{\mathrm{r}}=60.8 \mu \mathrm{s}$ and $t^{\mathrm{c}}=65.7 \mu \mathrm{s}$, respectively. The speed of the wave front and its time of arrival at A were estimated to be $c^{\mathrm{e}}=2.21 \mathrm{~mm} / \mu \mathrm{s}$ and $t_{\mathrm{A}}=54.3 \mu \mathrm{s}$, respectively.

\subsection{Results of impact tests and comparison with theory}

The impact velocities of the 120,240 and $360 \mathrm{~mm}$ PMMA strikers were estimated to be 9.5, 9.9 and $8.7 \mathrm{~m} / \mathrm{s}$, respectively. These velocities, with inaccuracy estimated to be within $\pm 5 \%$, were scaled with factors $0.98,0.97$ and 1.03 , respectively, in order to facilitate comparison of the strain pulse shapes. The experimental and theoretical results

for the strains $\varepsilon_{\mathrm{A}}, \varepsilon_{\mathrm{B}}$ and $\varepsilon_{\mathrm{C}}$ versus time $t$, and the corresponding spectrum $\left|\hat{\varepsilon}_{\mathrm{A}}\right|^{2}$ versus frequency $f$ are shown in Figs 8-10 for each striker. The time and frequency intervals have been limited to $0<t<1 \mathrm{~ms}$ and $0<f<60 \mathrm{kHz}$, as the strain amplitudes and their spectra become very small within these intervals.

The measured strains are filtered due to (i) the finite bandwidth $100 \mathrm{kHz}$ of the amplifiers and (ii) the finite length of the strain gauges. For the active gauge length used, $L_{\mathrm{g}}=6 \mathrm{~mm}$, and the estimated wave front speed $c^{\mathrm{e}}=2.21 \mathrm{~mm} / \mu \mathrm{s}$, the recorded strain at 
time $t$ becomes the time average of the strain at the centre of the gauge in the interval $\left(t-t_{\mathrm{g}} / 2, t+t_{\mathrm{g}} / 2\right)$, where $t_{\mathrm{g}}=L_{\mathrm{g}} / c^{\mathrm{e}}=2.71 \mu \mathrm{s}$ is the transit time for a wave front through the gauge. The resulting amplitude reduction is estimated to be $1.1 \mathrm{~dB}$ at 100 $\mathrm{kHz}$ and $0.4 \mathrm{~dB}$ at $60 \mathrm{kHz}$. Thus, at $100 \mathrm{~Hz}$ this amplitude reduction is significantly smaller than the $3 \mathrm{~dB}$ loss of gain of the amplifiers. Furthermore, the combined effect of the finite bandwidth and the finite gauge length is estimated to be quite small below 60 kHz.

It can be seen that there is a good general agreement between the experimental and the theoretical results. Thus, (i) there are only small deviations in pulse shapes which are mainly due to oscillations of the measured strains. Furthermore, (ii) the rise and fall of the measured main pulses are steep and their widths correspond to two transit times for the discontinuous viscoelastic wave front through the strikers, as predicted by theory.

Agreement according to (i) and (ii) requires that the conditions be close to $1 \mathrm{D}$ so that geometrical dispersion can be neglected. This means that the wave lengths $\lambda$ of the predominating waves must be much larger than the diameter $d$ of the striker and the bar, i.e. $\lambda \approx c^{\mathrm{e}} / f>>d$ or $f<<c^{\mathrm{e}} / d=185 \mathrm{kHz}$. In addition, agreement according to (ii) demands that the waves contain harmonic components which travel with speeds near the high-frequency limit $c=c^{\mathrm{e}}$ of the phase velocity. As pointed out, this occurs for $\omega=2 \pi f>>1 / t^{\mathrm{r}}$, i.e. at frequencies $f>>1 / 2 \pi t^{\mathrm{r}}=2.62 \mathrm{kHz}$. Thus, the predominating frequencies must be much lower than $185 \mathrm{kHz}$ (requirement for $1 \mathrm{D}$ conditions) at the same time as they must include frequencies much higher than $2.62 \mathrm{kHz}$ (requirement for steep rise and fall). Figures 8-10 show that these requirements are generally fulfilled. 


\section{Conclusions}

Some main conclusions from the case of viscoelastic impact studied can be summarised as follows: (i) The impact force consists of a main pulse that is either isolated or followed by a tail with finite or infinite length. The rise and fall of the main pulse are discontinuous, and the width of this pulse corresponds to two transit times through the striker for the viscoelastic wave front. (ii) The duration of contact (till first separation, if applicable) increases with $r$ from two transit times, first to a finite value and then jumps to infinity. In particular, the duration of viscoelastic impact may be infinite for values of $r$ smaller than unity. It is at least as long as that of elastic impact. (iii) For sufficiently small values of $r$, the striker rebounds without re-establishment of contact. For sufficiently large values of $r$, it stays in forceless contact with the bar after full transfer of its momentum. (iv) For intermediate values of $r$, contrary to the case of elastic impact, multiple contacts and separations may occur. (v) The momentum and energy transferred from the striker to the bar are at most as large as in elastic impact. (vi) The tail of the impact force and the phenomenon of multiple impacts can be avoided by choosing $r$ sufficiently small. This is of particular interest in SHPB testing. (vii) There is good agreement between experimental and theoretical results for strains in a PMMA bar impacted by strikers of the same material and with the same cross-sectional area. 


\section{References}

[1] De Saint-Venant B. Sur le choc longitudinal de deux barres élastiquues de grosseurs et de matières semblables ou différentes, et sur la proportion de leur force vive qui est perdue pour la translation ultérieure; Et généralement sur le movement longitudinal d'un système de deux ou plusieurs prismes élastiques. Journal de Mathématiques, 2e série 1867;12:237-376.

[2] De Saint-Venant B. Choc longitudinal de deux barres élastiques, dont l'une est extrêmement courte ou extrêmement roide par rapport à l'autre. Comptes Rendu 1868;66:650-653.

[3] Timoshenko S. Theory of Elasticity. McGraw-Hill Book Company, Inc., New York, Toronto, London 1951.

[4] Goldsmith W. Impact. Dover Publications, Inc., Mineola, New York 2001.

[5] Johnson W. Impact Strength of Materials. Edward Arnold Limited, London 1972.

[6] Gray III GT. Classic split Hopkinson pressure bar testing. ASM Handbook Vol 8, Mechanical testing and evaluation. ASM 2000:462-476.

[7] Fairhurst C. Wave mechanics of percussive drilling. Mine Quarry Engng $1961 ; 27: 122-30,169-78,327-28$.

[8] Lundberg B. Energy transfer in percussive rock destruction - I, II and III. Int J Rock Mech Min Sci \& Geomech. Abstr 1973;10:381-99,401-19,421-35.

[9] Gary G, Klepaczko JR, Zhao H. Generalization of split Hopkinson bar technique to use viscoelastic materials. Int J Impact Eng 1995; 16:529-530. 
[10] Gray III GT, Blumenthal WR. Split Hopkinson pressure bar testing of soft materials. ASM Handbook Vol 8, Mechanical testing and evaluation. ASM 2000:488-496.

[11] Lee E.H., Kanter I. Wave propagation in finite rods of viscoelastic material. Journal of Applied Physics 1953;24:1115-1122.

[12] Morrison JA. Wave propagation in rods of Voigt material and viscoelastic materials with three-parameter models. Quarterly of Applied Mathematics 1956;14:153-169.

[13] Zhao H. Analyse de l'Essai aux Barres d'Hopkinson: Application à la Mesure du Comportement Dynamique des Matériaux. PhD Thesis, l'Ecole Nationale des Ponts et Chaussées 1992.

[14] Zhao H, Gary G., Klepaczko. On the use of a viscoelastic split Hopkinson Pressure bar. Int. J. Impact Engng 1997;19:319-330.

[15] Kolsky H. Stress Waves in Solids. Dover Publications, Inc., New York 1963.

[16] Lundberg B, Lesser M. On impactor synthesis. J Sound Vibr 1978;58:5-14.

[17] Ödeen S, Lundberg B. Prediction of impact force by impulse response method. Int J Impact Eng 1991;11:149-158.

[18] Flugge. W. Viscoelasticity. Springer-Verlag, Berlin/Heidelberg 1975.

[19] Lundberg B, Blanc RH. Determination of mechanical material properties from the two-point response of an impacted linearly viscoelastic rod specimen. J Sound Vibr 1988;126:97-108.

[20] Blanc RH. Transient wave propagation methods for determining the viscoelastic properties of solids. Journal of Applied Mechanics 1993;60:763-768. 


\section{Captions}

Fig. 1. Axial impact between a cylindrical striker and a long cylindrical bar made of linearly viscoelastic materials.

Fig. 2. Experimental set-up.

Fig. 3. Impact force $F$ versus time $t$ for different striker-to-bar characteristic impedance ratios $r=Z_{1} / Z_{2}=A_{1} / A_{2}$. Comparison of elastic (thin curves) and viscoelastic (thick curves) impact. Relaxation time parameter $t^{\mathrm{r}}=0.5$ and creep time parameter $t^{\mathrm{c}}=0.75$. Unit of force $F_{1}^{\mathrm{e}}=(1 / 2) Z_{1}^{\mathrm{e}} V_{1}$ and unit of time $t_{1}^{\mathrm{e}}=2 L_{1} / c^{\mathrm{e}}$.

Fig. 4. Duration of contact $t_{0}$ of striker and bar (till the instant of first separation, if applicable) versus striker-to-bar characteristic impedance ratio $r=Z_{1} / Z_{2}=A_{1} / A_{2}$. Comparison of elastic (thin curves) and viscoelastic (thick curves) impact. Relaxation time parameter $t^{\mathrm{r}}=0.5$ and creep time parameter $t^{\mathrm{c}}=0.75$. Unit of time $t_{1}^{\mathrm{e}}=2 L_{1} / c^{\mathrm{e}}$. 
Fig. 5. Momentum $p_{2}$ transferred from striker to bar (till the instant of first separation, if applicable) versus striker-to-bar characteristic impedance ratio $r=Z_{1} / Z_{2}=$ $A_{1} / A_{2}$. Comparison of elastic (thin curves) and viscoelastic (thick curves) impact. Relaxation time parameter $t^{\mathrm{r}}=0.5$ and creep time parameter $t^{\mathrm{c}}=0.75$. Unit of momentum $p_{1}=m_{1} V_{1}$ and unit of time $t_{1}^{\mathrm{e}}=2 L_{1} / c^{\mathrm{e}}$.

Fig. 6. Energy $W_{2}$ transferred from striker to bar (till the instant of first separation, if applicable) versus striker-to-bar characteristic impedance ratio $r=Z_{1} / Z_{2}=A_{1} / A_{2}$. Comparison of elastic (thin curve) and viscoelastic (thick and dotted curves, the latter showing computed points) impact. Relaxation time parameter $t^{\mathrm{r}}=0.5$ and creep time parameter $t^{\mathrm{c}}=0.75$. Units of energy and time $W_{1}=(1 / 2) m_{1} V_{1}^{2}$ and $t_{1}^{\mathrm{e}}=2 L_{1} / c^{\mathrm{e}}$.

Fig. 7. Non-parametric (dots) and parametric (solid curves) complex modulus $E$ of the PMMA versus frequency $f=2 \pi / \omega$.

Fig. 8. Strain $\varepsilon$ versus time $t$ at strain gauge stations A, B and C and strain spectrum $|\hat{\varepsilon}|^{2}$ versus frequency $f$ at strain gauge station A of PMMA bar. Comparison of theory (thick curves) and experiment (thin curves) for $120 \mathrm{~mm}$ striker. Measured impact velocity scaled by factor 0.98 . 
Fig. 9. Strain $\varepsilon$ versus time $t$ at strain gauge stations A, B and C and strain spectrum $|\hat{\varepsilon}|^{2}$ versus frequency $f$ at strain gauge station A of PMMA bar. Comparison of theory (thick curves) and experiment (thin curves) for $240 \mathrm{~mm}$ striker. Measured impact velocity scaled by factor 0.97 .

Fig. 10. Strain $\varepsilon$ versus time $t$ at strain gauge stations A, B and C and strain spectrum $|\hat{\varepsilon}|^{2}$ versus frequency $f$ at strain gauge station A of PMMA bar. Comparison of theory (thick curves) and experiment (thin curves) for $360 \mathrm{~mm}$ striker. Measured impact velocity scaled by factor 1.03 . 
Figure 1

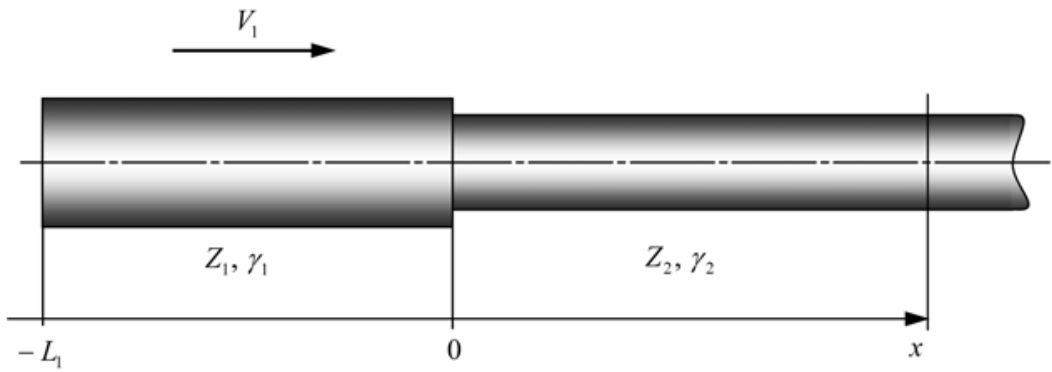


Figure 2

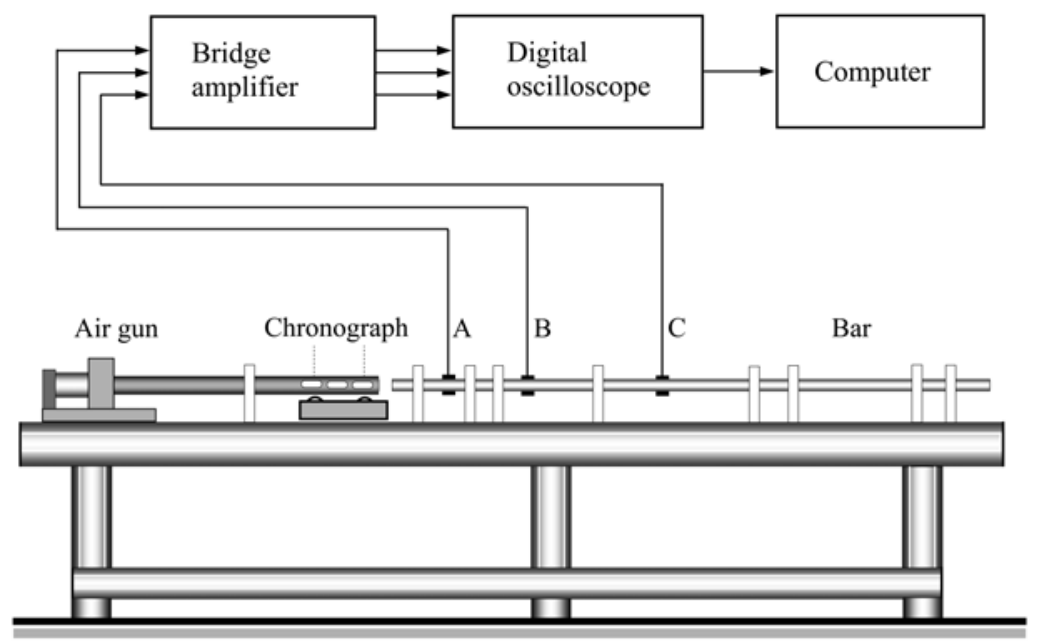


Figure 3
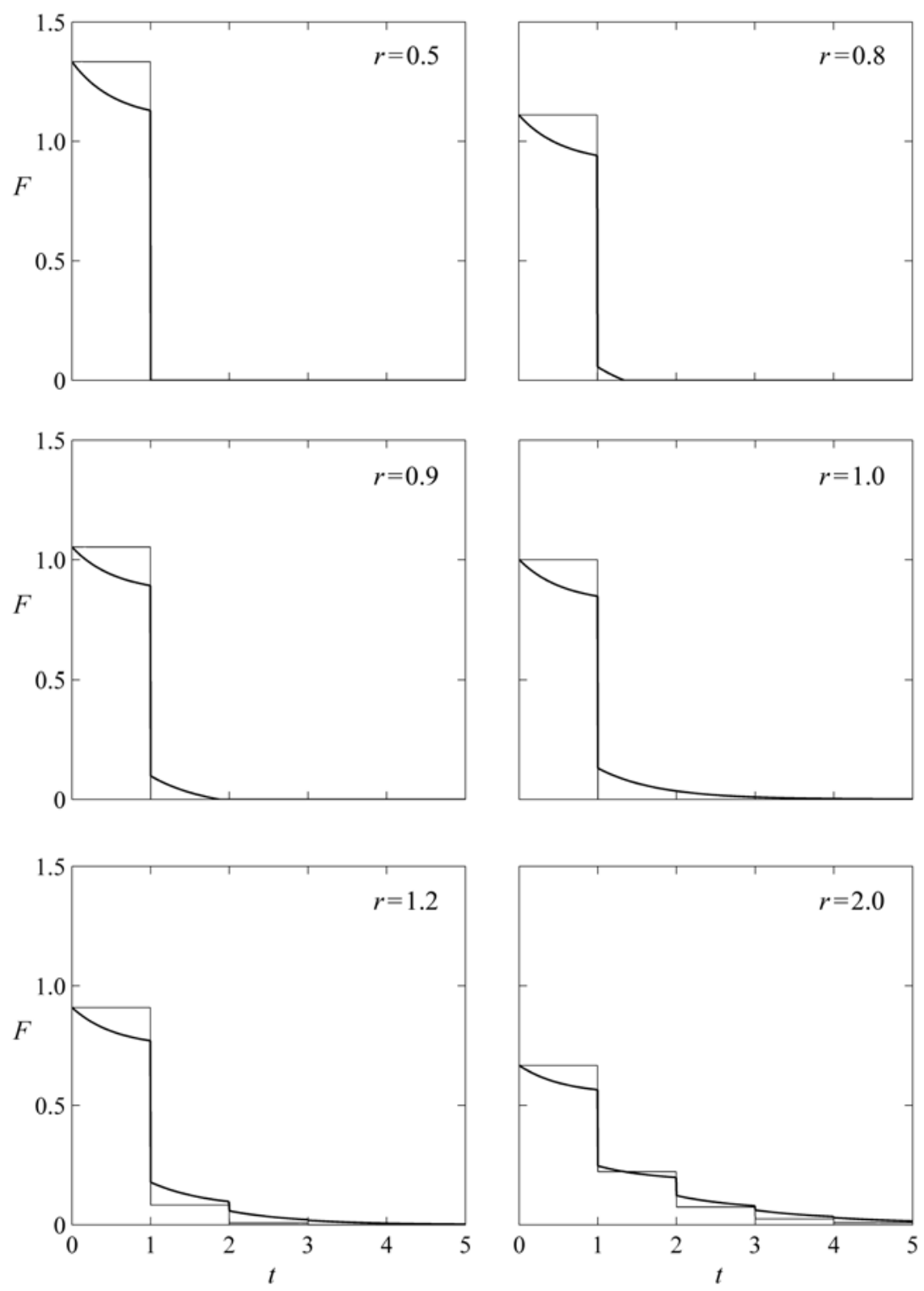
Figure 4

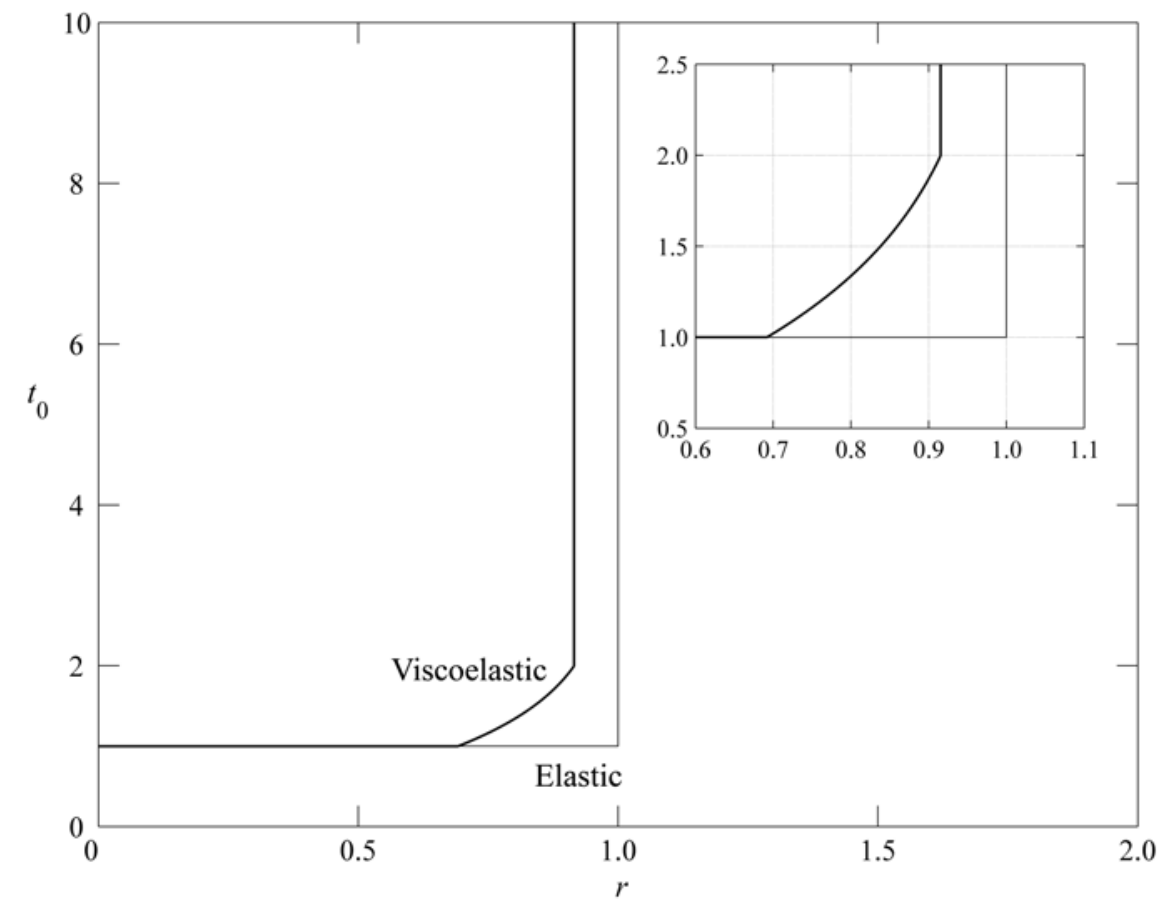


Figure 5

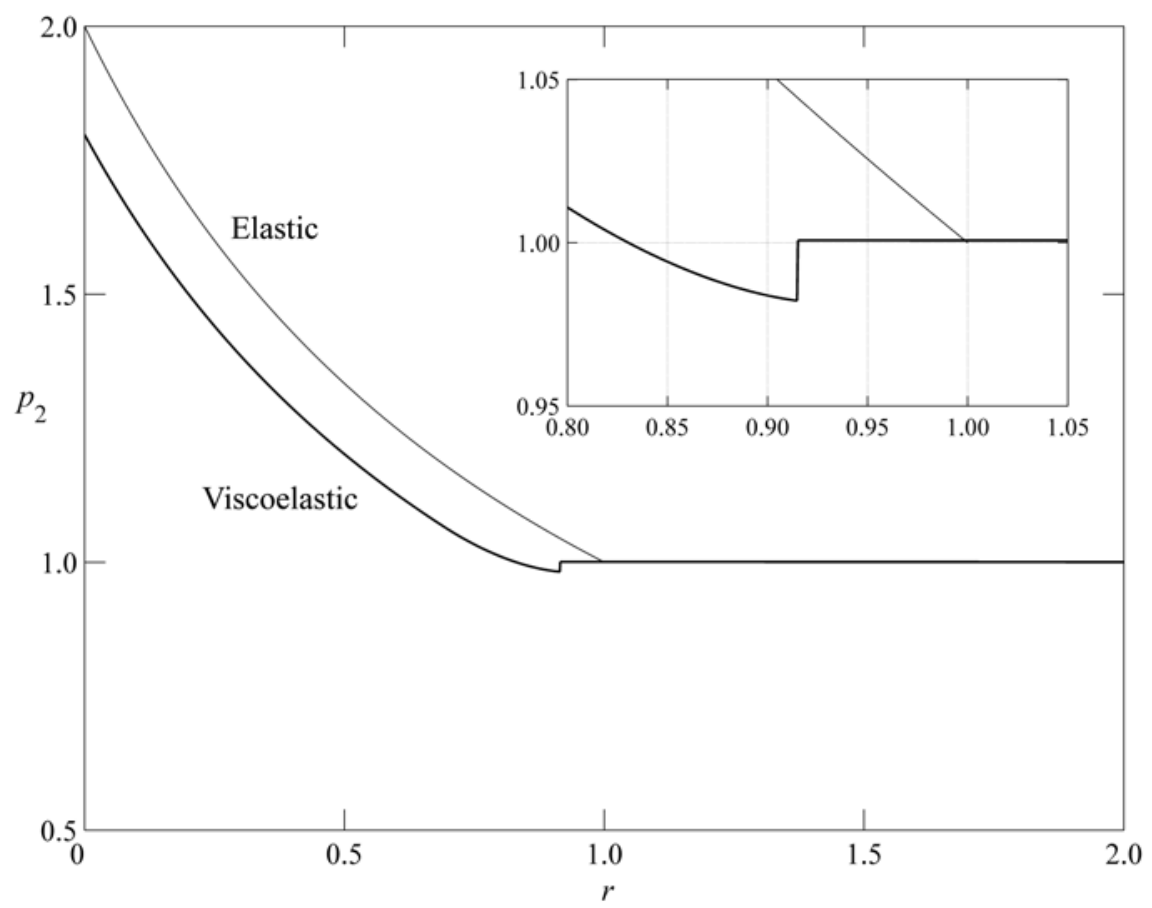


Figure 6

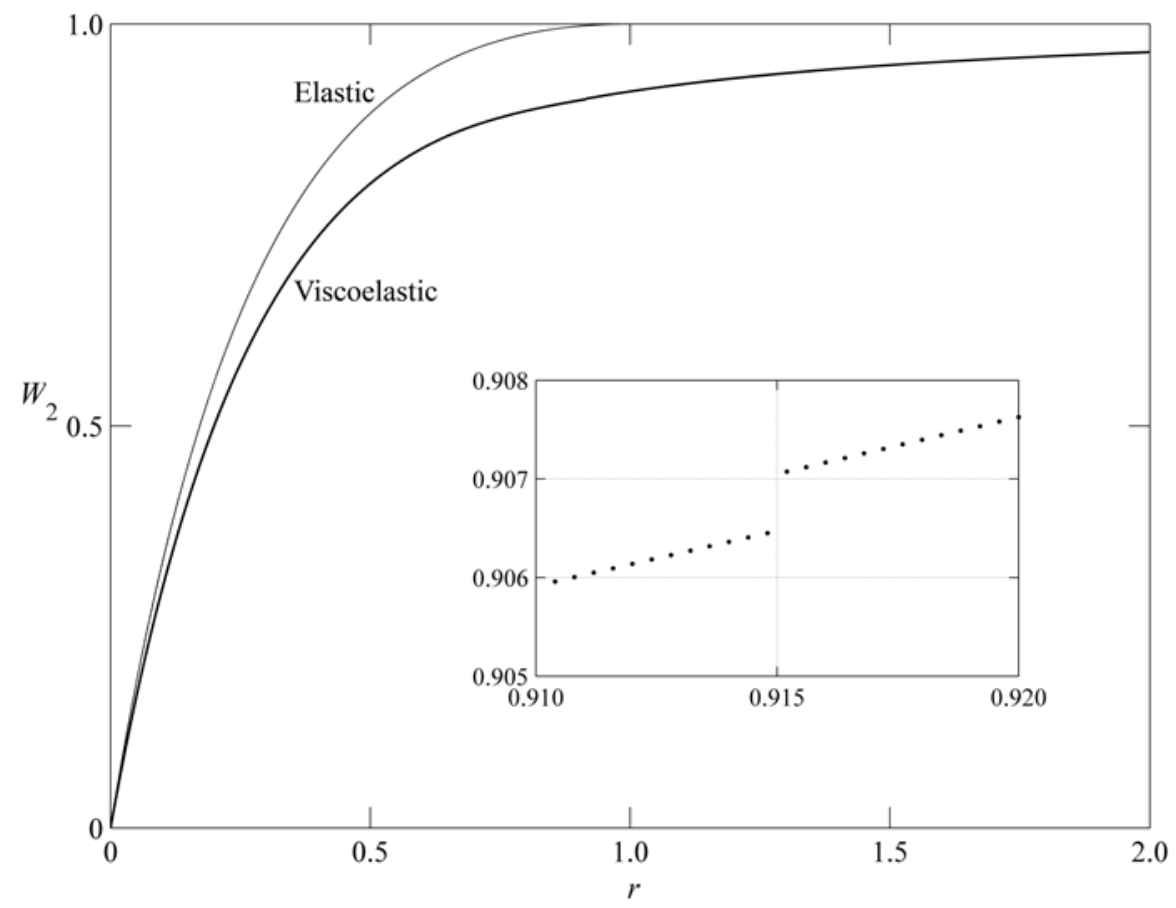


Figure 7

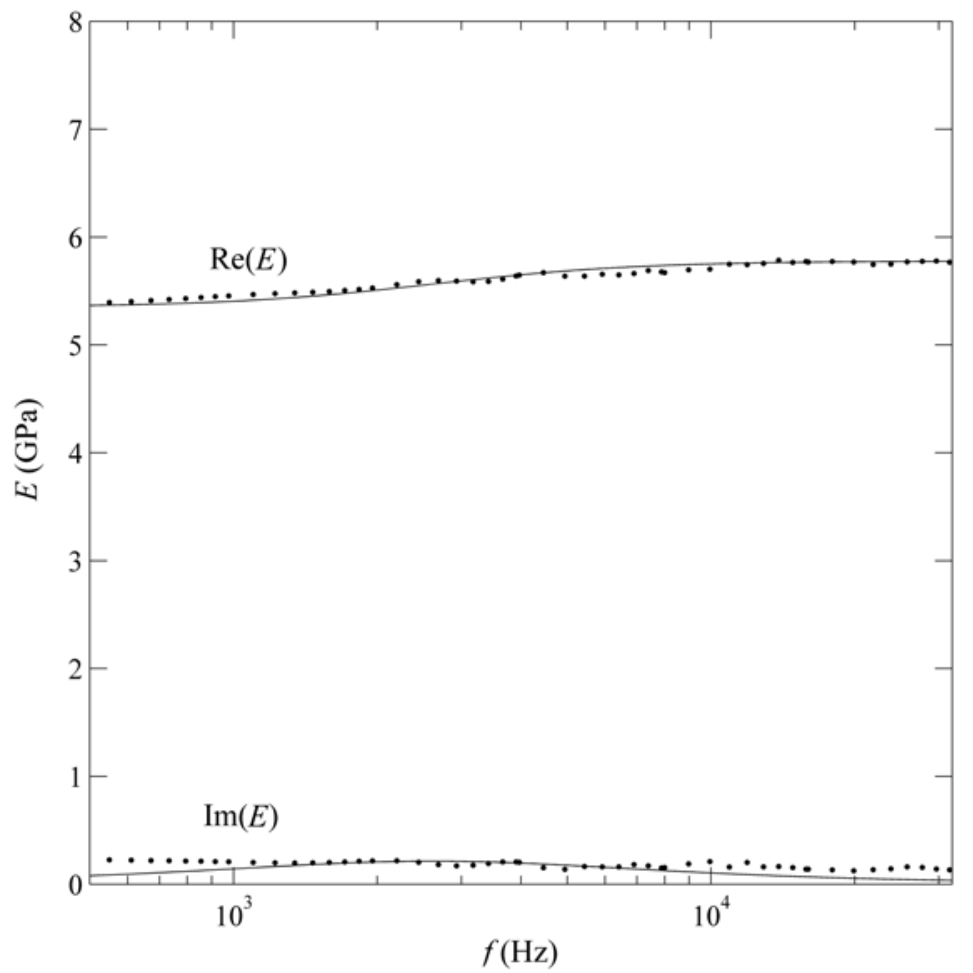


Figure 8
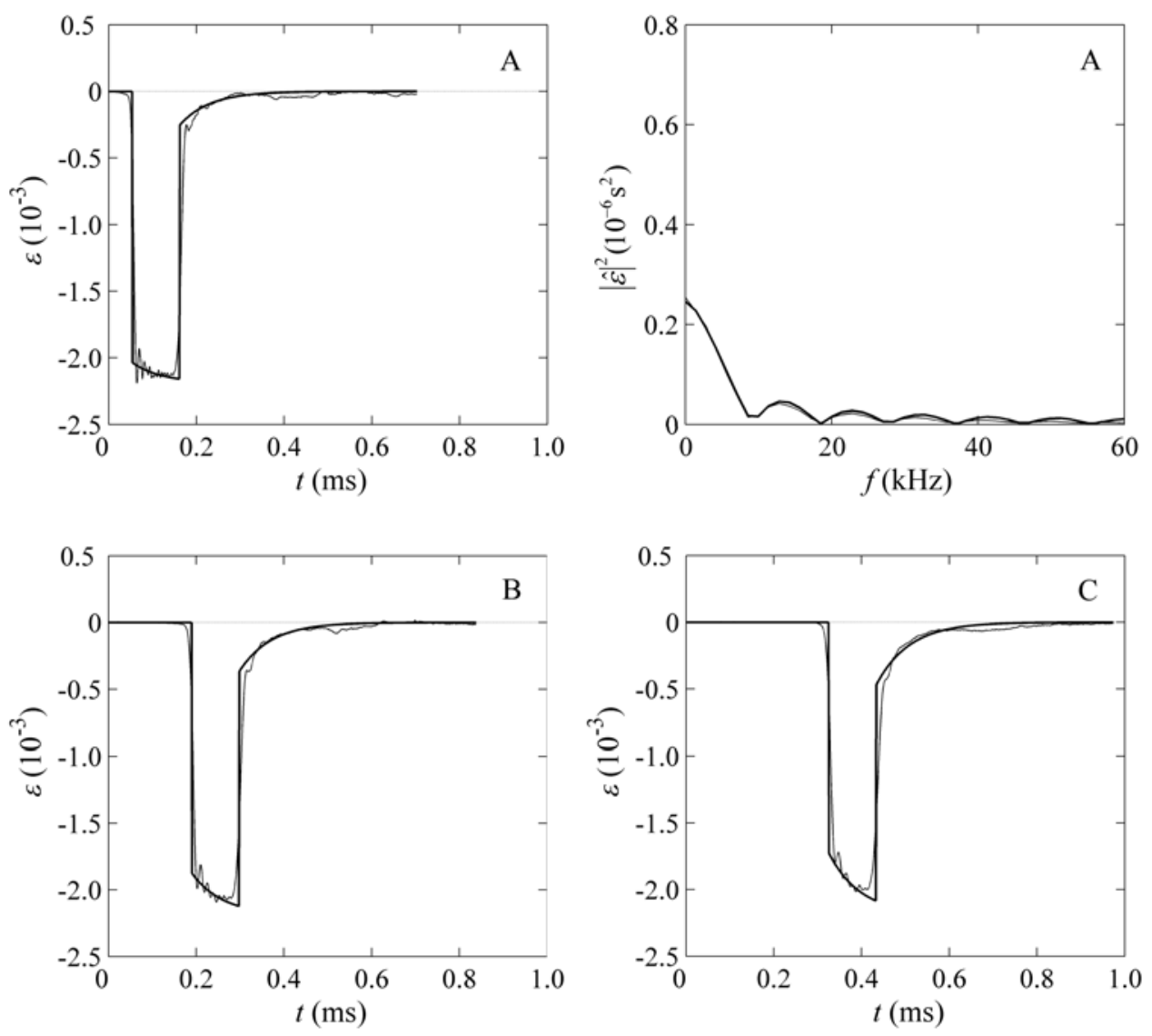
Figure 9
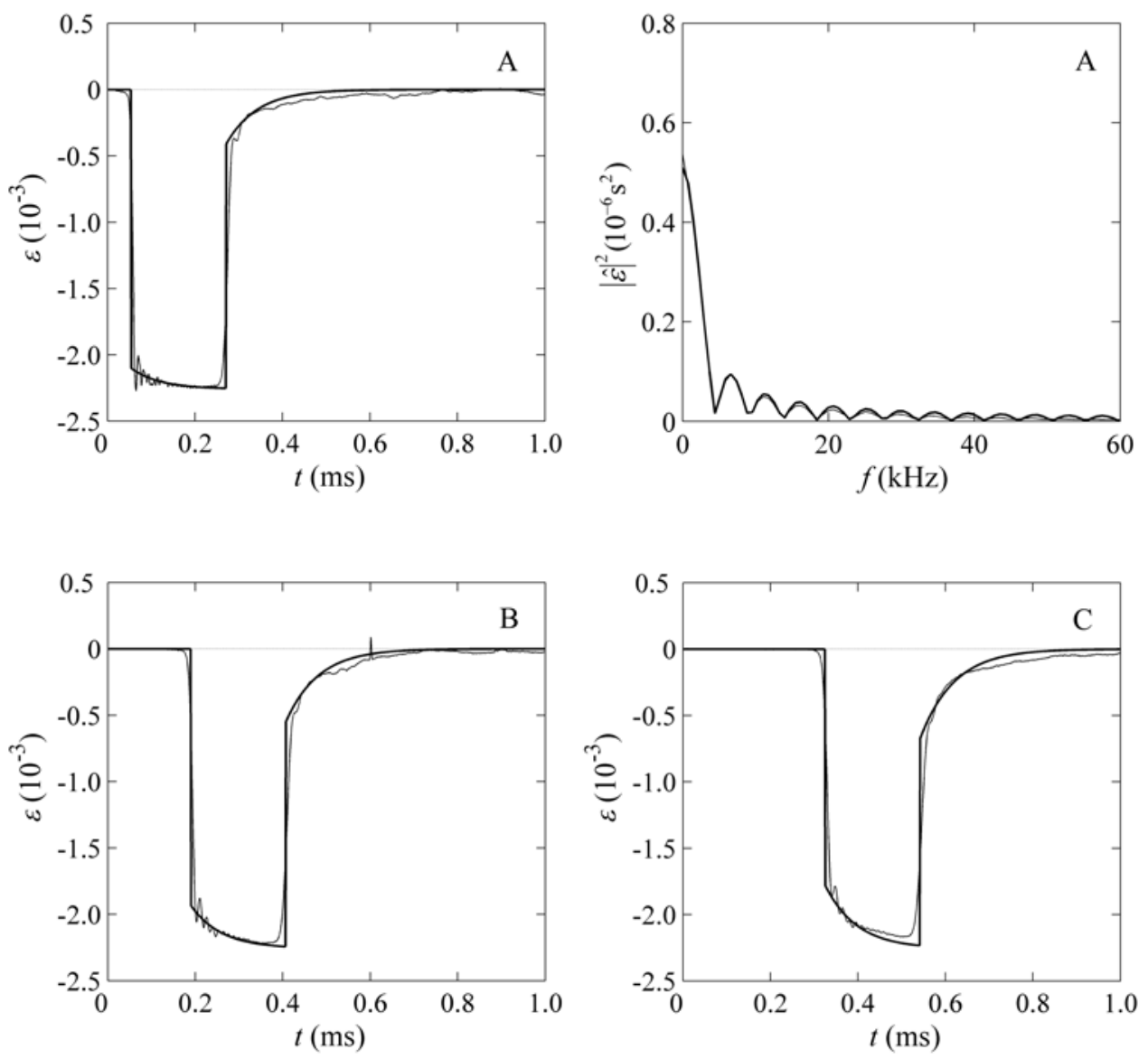
Figure 10
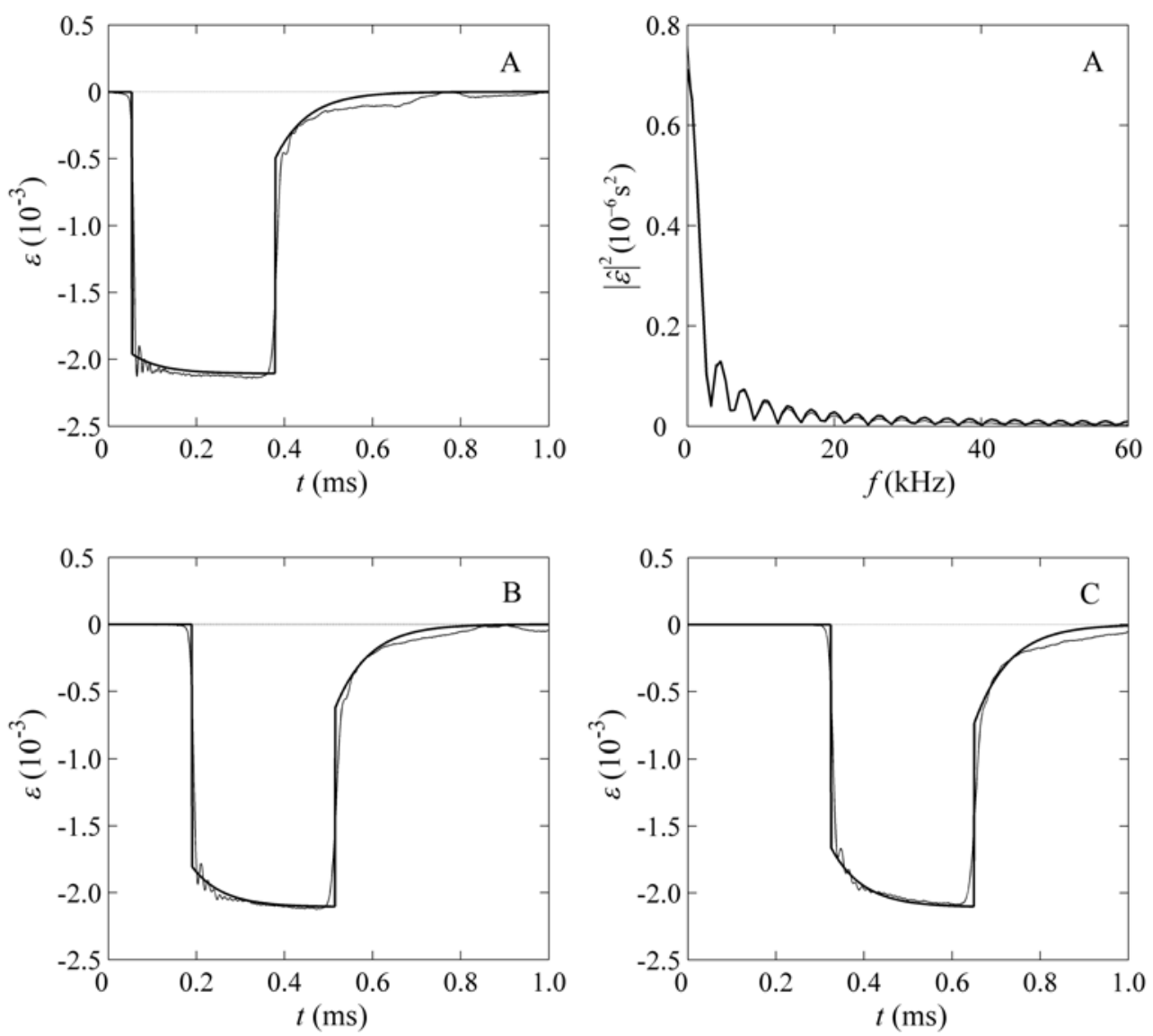